\title{
Zybert Light
}

Josef Zybert

Potential competing interests: The author(s) declared that no potential competing interests exist.

In Zybert Cosmology space is a fixed background.

Zybert Light has an Eigen Speed c, currently defined as $299792458 \mathrm{~m} / \mathrm{s}$.

Zybert Light has a Relative Speed $\hat{c}$, and $\hat{c}$ can be $<$ or $=$ or $>c$ or 0 .

Zybert Light has a Zybert Speed , the absolute speed.

Absolute speed means the speed relative to both fixed space and Zybertsphere centers fixed in fixed space.

The Eigen Speed is additive with the speed of the source giving its Zybert Speed .

The Zybert Speed is further changed, locally by any $M, M^{+}$or $M^{-}$, through an acceleration $G^{*} M / R^{\wedge} 2$, and, cosmically by $M$, $\mathrm{M}^{-}$or $\mathrm{M}^{+}$, through an acceleration $\mathrm{G}^{*} \mathrm{M} / \mathrm{R}^{\wedge} 2$ for the core and $(\mathrm{R} / \mathrm{Ro})^{*} \mathrm{G}^{*} \mathrm{M} / \mathrm{R} 0^{\wedge} 2$ for the cloud.

In the lab $\hat{c} \approx c$ but usually $\hat{c} \neq c$, mainly because there is always a $\Lambda$, no matter how small. 